\title{
Variable Stars in the Metal-Rich Globular Clusters NGC 6388 and NGC 6441
}

\author{
Barton Pritzl, Horace A. Smith \\ Dept. of Physics and Astronomy, Michigan State University, East \\ Lansing, MI 48824, USA \\ Márcio Catelan \\ Hubble Fellow, University of Virginia, Department of Astronomy, \\ P.O. Box 3818, Charlottesville, VA 22903, USA
}

\author{
Allen V. Sweigart \\ NASA/Goddard Space Flight Center, Laboratory for Astronomy and \\ Solar Physics, Code 681, Greenbelt, MD 20771, USA
}

\begin{abstract}
New observations show that RR Lyrae stars in the metal-rich globular clusters NGC 6388 and NGC 6441 have unusually long periods, which indicate that the horizontal branches of these clusters are unusually bright for their metallicities, as predicted by Sweigart \& Catelan (1998).
\end{abstract}

\section{Introduction}

The horizontal branches (HBs) in the metal-rich globular clusters NGC 6388 and NGC 6441 have prominent blue extensions not seen in other metal-rich globular clusters such as 47 Tuc (Rich et al. 1997). Most surprisingly, the HBs of these clusters slope upward with decreasing $B-V$ from the red clump to the top of the blue tail - an effect which cannot be caused by differences in age or mass loss and may therefore provide an important clue for understanding the origin of the second parameter effect. Sweigart \& Catelan (1998) have proposed several non-canonical scenarios for explaining the HB morphology. These scenarios predict that the HBs of NGC 6388 and NGC 6441 are unusually bright for their metallicity.

\section{RR Lyrae Stars and Cepheids}

Previous observations of RR Lyrae stars in NGC 6388 and NGC 6441 (Silbermann et al. 1994; Layden et al. 1999) implied that the HBs of the two globular clusters might indeed be unusually bright. We have obtained new CCD observations of NGC 6388 and NGC 6441 in order to construct improved light and color curves for previously known and newly discovered variables. The new photometry shows that RR Lyrae stars in these clusters are not coincident with field metal-rich RR Lyrae stars in the period-amplitude diagram. Instead, the NGC 6388 and NGC 6441 RR Lyrae stars occupy a portion of the period- 


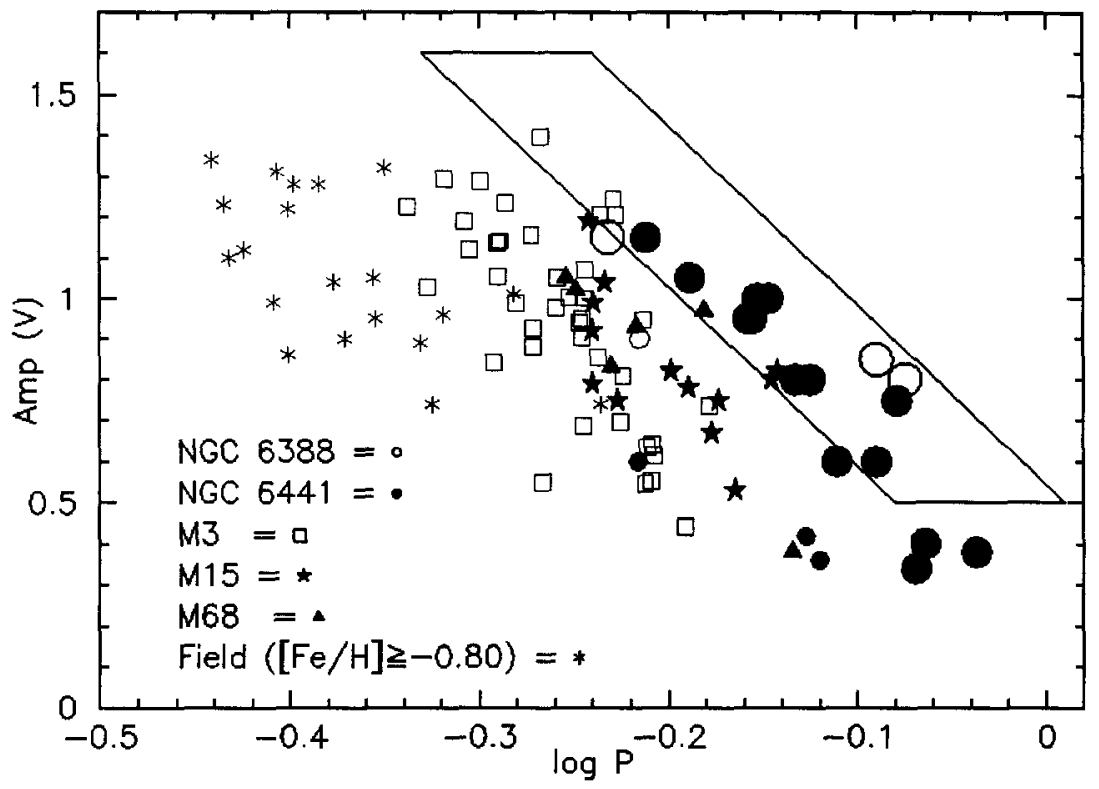

Figure 1. Period-amplitude diagram for RRab stars. The boxed area shows the predicted locations of RR Lyrae stars in helium-mixing models of Sweigart \& Catelan (1998).

amplitude diagram usually filled by RR Lyrae stars in metal-poor Oosterhoff II globular clusters. The mean periods of RRab stars in the two clusters are even longer than found in Oosterhoff II systems. This indicates that the RR Lyrae stars in NGC 6388 and NGC 6441 are among the brightest known, and that there is no unique correlation of metallicity and luminosity among RR Lyrae stars.

Four type II Cepheids have been discovered in the field of NGC 6388, making NGC 6388 the most metal-rich globular cluster known to contain type II Cepheids.

\section{References}

Layden, A. C., Ritter, L. A., Welch, D. L., \& Webb, T. M. A. 1999, AJ, 117, 1313

Rich, R. M., Sosin, C., Djorgovski, S. G., Piotto, G., King, I. R., Renzini, A., Phinney, E., Dorman, B., Liebert, J., \& Meylan, J. 1997, ApJ, 484, L25

Silbermann, N. A., Smith, H. A., Bolte, M., \& Hazen, M. L. 1994, AJ, 107, 1764 Sweigart, A. V. \& Catelan, M. 1998, ApJ, 501, L63 